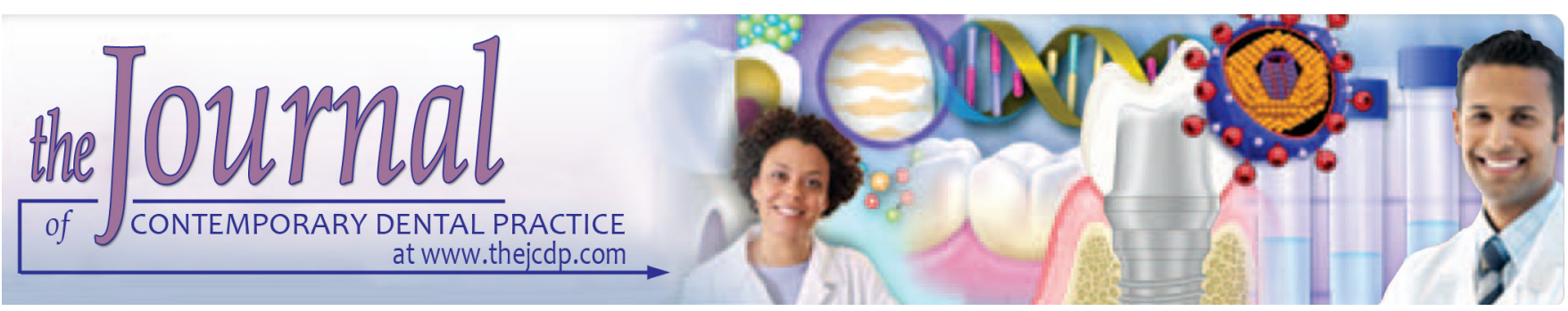

\title{
Melasma Associated with Periodontitis, Anemia, and Vitamin D Abnormalities: A Chance Occurrence or a Syndrome
}

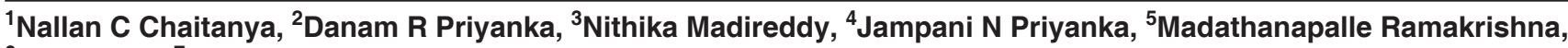

${ }^{6}$ Mujja Ajay, ${ }^{7}$ Ancy V Ignatius

\begin{abstract}
Aim: To correlate the possible association between melasma, periodontitis, vitamin $\mathrm{D}$ abnormalities, and anemia.

Materials and methods: A total of 192 subjects, of either gender within the age group of 30 to 70 years were divided equally into two groups, group I (case group, patients with melasma) and group II (control group, patients without melasma). In all the subjects, anemia and periodontitis were checked clinically, along with biochemical evaluation of serum vitamin $\mathrm{D}$ levels and hemoglobin $(\mathrm{Hb})$ percentage.
\end{abstract}

Results: Student's t-test was performed with group statistics and chi-square for intervariable correlation was carried out. A significant correlation was found between patients having melasma and serum vitamin D levels with a p-value of 0.050 . Between patients with melasma and periodontitis, there was a very significant correlation between the variables, with a p-value of 0.001 . Conversely, the correlation between melasma and $\mathrm{Hb}$ percentage was found to be insignificant with the $\mathrm{p}$-value 0.243 , and all of the $p$-values remained at $<0.05$. The study thus demonstrated significant correlation between melasma, vitamin $\mathrm{D}$ abnormalities, and clinical periodontitis ( $p$-value $<0.05$ ), and no significant correlation between melasma and $\mathrm{Hb}$.

Conclusion: The cross-sectional study revealed that patients with melasma may have abnormal serum vitamin $\mathrm{D}$ and clinical periodontitis. This presence may be considered a syndromic occurrence.

\footnotetext{
1-4,7Department of Oral Medicine and Radiology, Panineeya Mahavidyalaya Institute of Dental Sciences \& Research Centre Hyderabad, Telangana, India

${ }^{5}$ Department of Dentistry, Ministry of Health, Riyadh, Kingdom of Saudi Arabia

${ }^{6}$ Private Practitioner, Hyderabad, Telangana, India
}

Corresponding Author: Nallan C Chaitanya, Department of Oral Medicine and Radiology, Panineeya Mahavidyalaya Institute of Dental Sciences \& Research Centre, Hyderabad, Telangana, India, Phone: +91-8374930914, e-mail: nallanchaitanya@ gmail.com
Clinical significance: The present study was done to correlate and also evaluate periodontitis, vitamin D abnormalities, and anemia in patients with melasma. It is primarily an observational study, attempting to evaluate the co-occurrence of the abovementioned variables. The findings may prompt us to further investigate melasma for the presence of periodontitis, anemia, and vitamin $\mathrm{D}$ abnormalities.

Keywords: Hemoglobin percentage, Melasma, Periodontitis, Vitamin D.

How to cite this article: Chaitanya NC, Priyanka DR, Madireddy N, Priyanka JN, Ramakrishna M, Ajay M, Ignatius AV. Melasma Associated with Periodontitis, Anemia, and Vitamin D Abnormalities: A Chance Occurrence or a Syndrome. J Contemp Dent Pract 2018;19(10):1254-1259.

Source of support: Nil

Conflict of interest: None

\section{INTRODUCTION}

Melasma is a disorder, which is characterized by a bilateral hyperpigmentation of the facial skin confined to those areas of skin exposed to the sun. Chloasma or mask of pregnancy may be another form of this disorder. It may also be noticed in the neck and forearms. Prevalence is more common in women than in men. Three clinical patterns have been recognized based on their presentation: Malar, centrofacial and mandibular forms. Pathogenesis of melasma includes many risk factors like genetic predisposition, ultraviolet radiation, hormonal factors, and some contributing factors like oral contraceptives and drugs like phenytoin. ${ }^{1}$ Literature also reports abnormalities in the functioning of the thyroid gland in patients having melasma. Increased levels of luteinizing hormone and decreased levels of estradiol in serum with mild ovarian dysfunction were also reported in patients having melasma. ${ }^{2}$ 
Nutritional deficiencies and deficiency of vitamin $B_{12}$ reported skin pigmentation as a common manifestation. ${ }^{3}$ Although pigmentation of skin is affected by elevated serum iron levels, the occurrence of melasma in its deficiency is not clear. ${ }^{4}$

When the skin is exposed to ultraviolet radiation, it reacts with enzyme 7-dehydrocholesterol from where vitamin $\mathrm{D}_{3}$ (cholecalciferol) is synthesized by photochemical process in the skin and its active metabolite 1,25-dihydroxyvitamin $\mathrm{D}_{3}\left[1,25(\mathrm{OH})_{2} \mathrm{D}_{3}\right.$; calcitriol] is formed after a series of hydroxylation reactions first in the liver and then in the kidney. After the binding of $1,25(\mathrm{OH})_{2} \mathrm{D}_{3}$ to its intracellular receptor (vitamin D receptor, VDR), it functions like a hormone. The principal function of vitamin $\mathrm{D}$ is maintaining calcium homeostasis. Skin is the main target for the formation of vitamin D, as VDR is formed in cells of epithelium and melanocytes. Despite the active role of $1,25(\mathrm{OH})_{2} \mathrm{D}_{3}$ in the skin and use of vitamin $\mathrm{D}$ in the prevention and treatment of melanoma and psoriasis, no reports of melasma associated with vitamin $D$ reported have been reported so far. ${ }^{5}$

Periodontal disease may occur due to abnormal functioning of $1,25(\mathrm{OH})_{2} \mathrm{D}_{3}-\mathrm{VDR}$ system. The vitamin $\mathrm{D}$ deficiency can also lead to osteoporosis and chronic inflammatory diseases in addition to decreased mineral bone density. ${ }^{5}$ In the literature, there were no studies reporting the association between anemia and periodontitis, but there was a study which reported that anemia may predispose to periodontitis. ${ }^{6}$

\section{OBJECTIVES}

Till date, no studies in the literature have reported any sort of correlation between occurrence of melasma, periodontitis, serum vitamin $\mathrm{D}$ abnormalities, and $\mathrm{Hb}$ levels. The present cross-sectional observational study was done to estimate the presence of periodontitis, vitamin D abnormalities, and anemia in patients with melasma and to correlate and evaluate any possible association between each of them. The main objective of the present study is to correlate melasma with clinical evidence of periodontitis and levels of serum vitamin $\mathrm{D}$ and its co-occurrence with anemia. Thus, melasma on face may prompt to further investigate for the presence of periodontitis and anemia with vitamin $\mathrm{D}$ abnormalities.

\section{MATERIALS AND METHODS (BASED ON STROBE RECOMMENDATIONS)}

\section{Study Design}

The present study was approved by ethical clearance committee with IEC No. ECR/267/Indt/AP/2016 and was also entered in the National Clinical Trial Registry with number: NCT03241381.
A total of 192 subjects, of either gender, within the age group of 30 to 70 years were selected from outpatients attending the Department of Oral Medicine and Radiology, Panineeya Mahavidyala Institute of Dental Sciences and Research Centre, Hyderabad, Telangana, India. Informed consent was obtained from all the subjects included in the study. The subjects were categorized into two groups. Group I (case group) consisted of 96 subjects having melasma and group II (control group) consisted of 96 subjects without melasma. In all the subjects, anemia and periodontitis were checked clinically, along with biochemical evaluation of serum vitamin $\mathrm{D}$ levels and $\mathrm{Hb}$ percentage. About $2 \mathrm{~mL}$ of blood was collected from all the subjects for estimation of both serum vitamin $\mathrm{D}$ levels and $\mathrm{Hb}$ percentage.

\section{Study Setting and Participants}

The study was initiated in July 2017 and ended in Jan 2018 as the total sample size required for the study was obtained by then. A total of 192 subjects, who were willing to participate were taken into the study. Group I included 96 subjects with history of melasma and group II included 96 subjects without melasma.

The simple random sampling procedure of patient recruitment was followed with sample size determination based on prevalence of melasma in general population by the statistician. The exclusion criteria included the patients who did not want to be part of the study, who had disorders related to endocrine glands and with generalized pigmentation, and people on steroid therapy. There were no exclusions in the study after randomization. All the patients who gave consent for the study participated in it without any withdrawal in between.

\section{Variables, Data Sources, and Measurement}

Serum vitamin D analysis as laid down by the National Institutes of Health vitamin D fact sheet (2011) was used as template in both the groups by chemiluminescence methodology.

- $<12 \mathrm{ng} / \mathrm{mL}$ : Significant deficiency

- 12 to $20 \mathrm{ng} / \mathrm{mL}$ : Insufficiency

- $>20 \mathrm{ng} / \mathrm{mL}$ : Normal

- $50 \mathrm{ng} / \mathrm{mL}$ : Hypervitaminosis

- Regulations laid down by Russell's periodontal index status was used as the criteria to diagnose periodontitis clinically using mouth mirror and plain probe on all the gingival tissues circumscribing each tooth. Assessment was done for the inflammation of gingiva and loss of attachment with periodontal involvement.

- Examination of palpebral conjunctiva was done to check for pallor clinically and was correlated with serum $\mathrm{Hb}$ percentage in both the groups using photometry method. The normal levels of $\mathrm{Hb} \%$ were set at 14 to 
$16 \mathrm{gm} \%$ in males and 13 to $15 \mathrm{gm} \%$ in females. Any levels below these values were classified as anemic.

- Facial pigmentation in melasma was categorized as centrofacial, malar, and mandibular forms. Enquiry was done into menstrual changes every month along with oral contraceptive usage with relevant patients.

\section{Bias}

The study was carried out under the supervision of a periodontist and a dermatologist to confirm the findings within the groups. Melasma was evaluated for each patient and confirmed by the dermatologist. Each patient was evaluated by the periodontist who was the final authority to signify the presence of periodontitis in the patient clinically. The coordinators initially screened for melasma, anemia, and with available evidences of serum- vitamin D abnormalities, and these patients were referred to the respective dermatologist and physician for evaluation and treatment. Periodontal disease-affected patients were evaluated further and treated for the same by the periodontist. Radiographic criteria for the evaluation of periodontitis were not performed.

\section{Statistical Methods}

Comparison was done between both the groups for the presence or absence of three variables, i.e., periodontitis, serum vitamin $\mathrm{D}$ abnormalities, and serum $\mathrm{Hb}$ percentage with that of melasma.

The data obtained were tabulated and subjected to statistical analysis using Statistical Package for the Social Sciences, version VII software. Student's t-test was performed with group statistics and chi-square (Pearson correlation) for intervariable correlation was carried out.

\section{RESULTS}

Following results were obtained based on statistical analysis:

In both groups, the participants were analyzed for the serum analysis of vitamin $\mathrm{D}$ levels, $\mathrm{Hb}$ percentage, and clinical evidence of periodontitis.

\section{Gender and Age Distribution}

Gender characteristics showed that out of 96 patients with melasma, in group I, 10 were males and 86 were females. Similarly, out of 96 subjects in group II, 10 were males and 86 were females. Over all, in group I, the age ranged between 30 and 63 years and in group II, the age of the participants ranged between 30 and 65 years.

In group I, 26 patients were within the age group of 30 to 39 years, followed by 35 patients within the age group of 40 to 49 years, 31 patients within the age group of 50 to 59 years, and four patients within the age group of 60 to 69 years. The mean age of the study population under group I was 45.58 years.

In group II, 18 individuals were within the age group of 30 to 39 years, followed by 36 individuals within the age group of 40 to 49 years, 29 individuals within age group of 50 to 59 years, and three individuals within the age group of 60 to 69 years. The mean age of study population in group II was 48.24 years.

\section{Correlation between Melasma and Serum Vitamin D Levels, Hb Percentage and Clinical Periodontitis (Student's t-test)}

A significant correlation was found between patients having melasma and serum vitamin $\mathrm{D}$ levels with a p-value of 0.050. Between patients with melasma and periodontitis, there was a very significant correlation between the variables, with the p-value of 0.001 . Conversely, the correlation between melasma and $\mathrm{Hb}$ percentage was found to be insignificant with the p-value 0.243 , and all of the p-values remained at $<0.05$.

\section{Outcome Data: Intergroup Comparisons}

\section{Serum Vitamin D Characteristics (Table 1)}

In group $\mathrm{I}$, serum vitamin D levels were normal in 30 patients (31.3\%), deficient in 32 patients (33.3\%), insufficient in 33 patients $(34.4 \%)$, and in 1 patient $(1.0 \%)$, there was hypervitaminosis. In group II, the levels of vitamin D were normal in 42 patients $(43.8 \%)$, deficient in 17 patients $(17.7 \%)$, and insufficient in 37 patients (38.5\%). Mean values of vitamin D levels in group I were 19.4 and in the control, these were 20.6. The mean serum vitamin $\mathrm{D}$ values were higher in patients having melasma when compared with the controls with 12.06 and 9.4 respectively. It would be noteworthy to mention that the individuals with serum vitamin D insufficiency were found to be maximum in group II (controls) than in group I (cases).

Pearson correlation revealed the vitamin D abnormalities, i.e., hypervitaminosis, insufficiencies, and

Table 1: Intervariable correlation of vitamin D abnormalities with melasma

\begin{tabular}{|c|c|c|c|c|c|c|c|}
\hline \multirow{4}{*}{$\begin{array}{l}\text { Levels of } \\
\text { vitamin } D\end{array}$} & \multicolumn{6}{|c|}{ Vitamin D group cross-tabulation } & \multirow[b]{4}{*}{ p-value } \\
\hline & \multicolumn{4}{|c|}{ Groups } & & & \\
\hline & \multicolumn{2}{|c|}{ Case } & \multicolumn{2}{|c|}{ Control } & \multicolumn{2}{|c|}{ Total } & \\
\hline & Count & $(\%)$ & Count & $(\%)$ & Count & $(\%)$ & \\
\hline Normal & 30 & 31.3 & 42 & 43.8 & 72 & 37.5 & $0.050^{*}$ \\
\hline Deficiency & 32 & 33.3 & 17 & 17.7 & 49 & 25.5 & \\
\hline Hyper & 1 & 1 & 0 & 0.0 & 1 & 0.5 & \\
\hline Insufficiency & 33 & 34.4 & 37 & 38.5 & 70 & 36.5 & \\
\hline Total & 96 & 100 & 96 & 100 & 192 & 100 & \\
\hline Chi square & 7.820 & & & & & & \\
\hline
\end{tabular}

*Significant at $p<0.05$ 
Melasma Associated with Periodontitis, Anemia, and Vitamin D Abnormalities

Table 2: Intervariable correlation of $\mathrm{Hb}(\%)$ with melasma

\begin{tabular}{|c|c|c|c|c|c|c|c|}
\hline \multirow[b]{4}{*}{$\mathrm{Hb} \%$} & \multicolumn{6}{|c|}{ CBP group cross-tabulation } & \multirow[b]{4}{*}{$p$-value } \\
\hline & \multicolumn{4}{|c|}{ Groups } & \multirow{2}{*}{\multicolumn{2}{|c|}{ Total }} & \\
\hline & \multicolumn{2}{|c|}{ Cases } & \multicolumn{2}{|c|}{ Controls } & & & \\
\hline & Count & (\%) & Count & (\%) & Count & (\%) & \\
\hline Normal & 51 & 53.1 & 59 & 61.5 & 110 & 57.3 & 0.243 \\
\hline Anemia & 45 & 46.9 & 37 & 38.5 & 82 & 42.7 & \\
\hline Total & 96 & 100.0 & 96 & 100.0 & 192 & 100.0 & \\
\hline Chi-square & 1.362 & & & & & & \\
\hline
\end{tabular}

deficiencies in particular; when correlated with melasma, there was a statistically significant correlation $(0.050)$ between them.

\section{Serum Hb Percentage Characteristics (Table 2)}

In group $\mathrm{I}, \mathrm{Hb}$ percentage was normal in 51 patients $(53.1 \%)$ and 45 patients $(46.9 \%)$ were anemic, whereas in group II, $\mathrm{Hb}$ percentage was normal in 59 patients $(61.5 \%)$ and 37 patients $(38.5 \%)$ were anemic. Mean values of $\mathrm{Hb}$ levels in group I were 11.57 and in group II, these were 12.22. The mean $\mathrm{Hb}$ percentages were higher in controls when compared with the patients having melasma.

Comparison between melasma and $\mathrm{Hb}$ percentage by Chi-square test revealed that there was no statistically significant correlation of $\mathrm{p}$-value 0.243 between them.

\section{Melasma-Periodontitis Characteristics (Table 3)}

In group I, 68 (70.8\%) patients suffered from periodontitis and $28(29.2 \%)$ patients had no clinical evidence of periodontitis, whereas in group II, $27(28.1 \%)$ patients suffered from periodontitis and $69(71.9 \%)$ patients had no clinical evidence of periodontitis.

There was good statistical significance between melasma and periodontitis with a p-value 0.001 .

\section{DISCUSSION}

This study was done to report the presence of periodontitis, vitamin $\mathrm{D}$ abnormalities, and anemia in patients with melasma in a cross-section of population using controls. In addition, chi-square test for intervariable correlation was carried out to evaluate whether they occurred coincidentally or had any correlation. In this study, there was a significant correlation between patients having melasma and serum vitamin D abnormalities ( $p$-value 0.050). Various factors may be implicated which could have contributed to their occurrence together. After the binding of $1,25(\mathrm{OH})_{2} \mathrm{D}_{3}$ to its intracellular receptor (VDR), it shows its hormonal activity. Skin is the target for vitamin D production, as VDR is also expressed in cells of epithelium and melanocytes. ${ }^{5-7}$ Studies reported the increased tyrosinase activity and associated decrease in cell proliferation in melanoma cells in vitro and in vivo
Table 3: Intervariable correlation of periodontitis with melasma

\begin{tabular}{|c|c|c|c|c|c|c|c|}
\hline \multirow{3}{*}{$\begin{array}{l}\text { Clinical } \\
\text { periodontitis }\end{array}$} & \multicolumn{4}{|c|}{ Groups } & \multirow{2}{*}{\multicolumn{2}{|c|}{ Total }} & \multirow[b]{3}{*}{$p$-value } \\
\hline & \multicolumn{2}{|c|}{ Cases } & \multicolumn{2}{|c|}{ Controls } & & & \\
\hline & Count & (\%) & Count & (\%) & Count & (\%) & \\
\hline Present & 68 & 70.8 & 27 & 28.1 & 95 & 49.5 & $0.001^{*}$ \\
\hline Absent & 28 & 29.2 & 69 & 71.9 & 97 & 50.5 & \\
\hline Total & 96 & 100.0 & 96 & 100.0 & 192 & 100.0 & \\
\hline Chi-square & 35.025 & & & & & & \\
\hline
\end{tabular}

*Significant at $p<0.05$

in response to $1,25(\mathrm{OH})_{2} \mathrm{D}_{3}$, which binds with similar affinity to human melanocytes. ${ }^{8}$ Ranson et al $^{7}$ had found that there was similar stimulatory activity in melanocytes of normal skin. ${ }^{9}$ However, some studies contraindicated that vitamin D has no effect on the melanization of cultured human melanocytes. ${ }^{10}$ Melanin in the skin plays a prominent role in synthesis of vitamin $D$ which absorbs and scatters ultraviolet radiation- $\mathrm{B}$, making conversion of 7-dehydrocholesterol to previtamin $\mathrm{D}_{3}$ which showed slower vitamin $\mathrm{D}$ synthesis in dark-skinned individuals than in light-skinned ones. ${ }^{11}$ Through VDR activation, vitamin D plays a significant role in the process of anticancer mechanism, immunomodulatory action, cardiovascular, and musculoskeletal health. ${ }^{5}$

A recent study reported that there is a significant association between serum vitamin $\mathrm{D}$ abnormalities and periodontitis, as the intake of vitamin $\mathrm{D}$ and calcium showed improvement in periodontal health. ${ }^{12}$ A study conducted by reported that a higher dose of 800 to 1000 IU of vitamin D supplementation reduced the severity of periodontal disease. Vitamin D not only had direct effects on bone but also had antibiotic effects on pathogens leading to periodontitis and anti-inflammatory effects by inhibiting inflammatory cytokines that lead to periodontal destruction. ${ }^{13,14}$ In the present study, there was significant alteration in vitamin D levels among patients who suffered from periodontitis. Additionally, such patients also had melasma on their face which invariably had significant statistical correlation among all the three variables, i.e., vitamin $\mathrm{D}$ abnormalities, melasma, and periodontitis.

Several studies reported development of melasma in post-menopausal women who are under hormonal replacement therapy (HRT) and oral contraceptive pills. Johnston et al. ${ }^{15}$ reported that a 54 -year-old woman developed melasma on forearms on taking HRT for 8 years. Older and postmenopausal women who are on supplementary estrogen therapy commonly developed melasma on forearms, which may be due to the presence of estrogen-sensitive melanocytes in the forearm, which mature at later stages and these individuals are reported to have facial melasma at younger age. ${ }^{16}$

The role of female hormones in onset of melasma indicated the correlation with menstrual periods and 
higher prevalence among the women on exogenous estrogen and/or progesterone. ${ }^{17}$ Melasma of face was observed with increase in estrogen levels. ${ }^{18-20}$ The face is the area, which is most likely to predispose to melasma as the estrogens increase the vascularization of the skin and suppress sebaceous gland activity. ${ }^{21}$

Studies done by Lainson et al., ${ }^{22}$ Hutter et al., ${ }^{23}$ Gokhale et al., ${ }^{24}$ Naik et al., ${ }^{25}$ Aljohani, ${ }^{26}$ and Wakai et al. ${ }^{27}$ found no correlation between $\mathrm{Hb}$ levels and periodontal status. Anemia may be noticed in the individuals in periodontitis patients due to the release of proinflammatory cytokines, which lead to decreased blood counts by downregulation of erythropoiesis in the bone marrow. ${ }^{23,24}$ However, studies showing association between the melasma as a cofactor and anemia were not attempted so far. In this study, there was no significant correlation between melasma and $\mathrm{Hb} \%$ abnormalities.

However, it has to be emphasized that when intervariable vitamin $\mathrm{D}$ abnormalities, such as deficiency, excess, and insufficiency were evaluated with melasma, there were no encouraging results. Hence, inference was drawn in a way that melasma, vitamin D abnormalities, and periodontitis exist in the population with no anemia. A study had hypothesized that vitamin D abnormalities could cause anemia due to the inadequate production of erythropoietin by developing a persistent inflammatory state, which leads to overproduction of hepcidin by decreasing erythroid production. ${ }^{28}$ Another study reported that anemia may predispose to vitamin $\mathrm{D}$ deficiency as they are less likely to go outdoors and experience sun exposure and due to anemia, they become easily fatigued, and stated that several other confounding factors were involved in their association. ${ }^{29}$ However, in this study, there was no statistical significance among patients having melasma and anemia (p-value 0.243).

\section{INFERENCE AND CONCLUSION}

In this study, there was a significant correlation between patients having melasma, vitamin $\mathrm{D}$ abnormalities, and periodontitis, but there was no significance among patients having melasma and anemia. As such, this combination is rare and unreported. This presence may be considered a syndromic occurrence or coincidence which is to be further investigated. It may be attributed to as a syndromic association as "Nallan C-Melasma Syndrome" having a triad of melasma, clinical periodontitis, and vitamin D abnormalities.

\section{LIMITATIONS}

The finding in the study could have reflected more accurately if detailed blood parameters in terms of specific deficiency factor were checked. Calcium levels were not investigated taking into account the cost issue while estimating vitamin D serum levels. Different forms of melasma were not evaluated specifically correlating with other parameters. Only the presence and absence were taken into consideration. Periodontitis was not evaluated radiographically; only clinical examination was done which might have led to inappropriate assessment of periodontitis that may have been clinically evident but radiographically absent. Interobserver variability could have given unbiased results during clinical examination for periodontitis. Thus, further studies with samples focusing on variants of melasma, periodontitis, and vitamin D abnormalities, which could overcome the above flaws for effective assessment are recommended.

\section{ACKNOWLEDGMENTS}

Authors would like to acknowledge the staff and postgraduate student, Dr Kondaiah, Department of Oral Medicine and Radiology, Panineeya Dental College, Hyderabad, Telangana, India for helping them during the entire period of the study.

\section{REFERENCES}

1. Bagherani N, Gianfaldoni S, Smoller B. An overview on melasma. J Pigment Disord 2015 Sep;2:216.

2. Sarkar R, Arora P, Garg VK, Sonthalia S, Gokhale N, Melasma update. Indian Dermatol Online J 2014 Oct;5(4):426-435.

3. Najad SB, Khodaiiani E, Herizchi H, Mehrabi P. Frequency of iron deficiency anemia, folate and vitamin B12 deficiency in patients with melasma. Med J Tabriz Univ Med Sci 2012 Jun-Jul;34(2):1-8.

4. Behrangi E, Baniasadi F, Esmaeeli S, Hedayat K, Goodarzi A, Azizian Z. Serum iron level, ferritin and total iron binding capacity level among nonpregnant women with and without melasma. J Res Med Sci 2015 Mar 20(3):281-283.

5. Anand N, Chandrasekaran SC, Rajput NS. Vitamin D and periodontal health: current concepts. J Indian Soc Periodontol 2013 May;17(3):302-308.

6. Nair SK, Faizuddin M, Jayanthi D. Anemia and periodontitis: an enigma? IOSR J Dent Med Sci 2013 Nov-Dec;11(4):7178.

7. Ranson M, Posen S, Mason RS. Human melanocytes as a target tissue for hormones: in vitro studies with 1 alpha-25, dihydroxyvitamin D3, alphamelanocyte stimulating hormone, and beta-estradiol. J Invest Dermatol 1988 Dec;91(6):593-598.

8. Milde P, Hauser U, Simon T, Mall G, Ernst V, Haussler MR, Frosch P, Rauterberg EW. Expression of 1,25-dihydroxyvitamin D3 receptors in normal and psoriatic skin. J Invest Dermatol 1991 Aug;97(2):230-239.

9. Kuroki T. Possible functions of 1 alpha,25-dihydroxyvitamin D3, an active form of vitamin D3, in the differentiation and development of skin. J Invest Dermatol 1985 Jun;84(6):459-460.

10. Mansur CP, Gordon PR, Ray S, Holick MF, Gilchrest BA. Vitamin D, its precursors, and metabolites do not affect melanization of cultured human melanocytes. J Invest Dermatol 1988 Jul;91(1):16-21. 
11. Jablonski NG. The evolution of human skin and skin color. Annu Rev Anthropol 2004 Oct;33:585-623.

12. Andresen C, Olson E, Nduaka CI, Pero R, Bagi CM. Action of calciotropic hormones on bone metabolism - role of vitamin D3 in bone remodeling events. Am J Immunol 2006 Feb;2(2):40-51.

13. Cozzolino M, Lu Y, Finch J, Slatopolsky E, Dusso AS. p21WAF1 and TGF-alpha mediate parathyroid growth arrest by vitamin D and high calcium. Kidney Int 2001 Dec;60(6):2109-2117.

14. Zittermann A. Vitamin D in preventive medicine: are we ignoring the evidence? Br J Nutr 2003 May;89(5):552-572.

15. Johnston GA, Sviland L, McLelland J. Melasma of the arms associated with hormone replacement therapy. Br J Dermatol 1998 Nov;139(5):932.

16. O'Brien TJ, Dyall-Smith D, Hall AP. Melasma of the forearms. Australas J Dermatol 1997 Feb;38(1):35-37.

17. Parmley $\mathrm{T}, \mathrm{O}^{\prime}$ Brien TJ. Skin changes during pregnancy. Clin Obstet Gynecol 1990 Dec;33(4):713-717.

18. Snell RS. The pigmentary changes occurring in the breast skin during pregnancy and following estrogen treatment. J Invest Dermatol 1964 Sep;43:181-186.

19. Im S, Lee ES, Kim W, On W, Kim J, Lee M, Kang WH. Donor specific response of estrogen and progesterone on cultured human melanocytes. J Korean Med Sci 2002 Feb;17(1):58-64.

20. Lieberman R, Moy L. Estrogen receptor expression in melasma: results from facial skin of affected patients. J Drugs Dermatol 2008 May;7(5):463-465.

21. Sheth VM, Pandya AG Melasma: a comprehensive update: part I. J Am Acad Dermatol 2011 Oct;65(4):689-697.
22. Lainson PA, Brady PP, Fraleigh CM. Anemia, a systemic cause of periodontal disease? J Periodontol 1968 Jan;39(1): 35-38.

23. Hutter JW, van der Velden U, Varoufaki A, Huffels RA, Hoek FJ, Loos BG. Lower numbers of erythrocytes and lower levels of haemoglobin in periodontitis patients compared to control subjects. J Clin Periodontol 2001 Oct;28(10):930-936.

24. Gokhale SR, Sumanth S, Padhye AM. Evaluation of blood parameters in patients with chronic periodontitis for signs of anemia. J Periodontol 2010 Aug;81(8):1202-1206.

25. Naik V, Acharya A, Deshmukh VL, Shetty S, Shirhatti R. Generalized, severe, chronic periodontitis is associated with anemia of chronic disease: a pilot study in urban Indian males. J Invest Clin Dent 2010 Nov;1(2):139-143.

26. Aljohani HA. Association between hemoglobin level and severity of chronic periodontitis. JKAU Med Sci 2009 Dec;17(1):53-64.

27. Wakai K, Kawamura T, Umemura O,Hara Y, Machida J, Anno T, Ichihara Y, Mizuno Y, Tamakoshi A, Lin Y, et al. Associations of medical status and physical fitness with periodontal disease. J Clin Periodontol 1999 Oct;26(10):664-672.

28. Sim JJ, Lac PT, Liu ILA, Meguerditchian SO, Kumar VA, Kujubu DA, Rasgon SA. Vitamin D deficiency and anemia: a cross-sectional study. Ann Hematol 2010 May;89(5):447-452.

29. Monlezun DJ, Camargo CA Jr, Mullen JT, Quraishi SA. Vitamin D status and the risk of anemia in community-dwelling adults: results from the National Health and Nutrition Examination Survey 2001-2006. Medicine (Baltimore) 2015 Dec;94(50): e1799. 